\title{
Errors of the Intellect: A neglected aspect in teaching
}

\author{
Balakrishna Palanki \\ Mahaveer Educational Society, Bandlaguda, Keshavgiri Post, Hyderabad 500002, India \\ palankibalakrishna@yahoo.com
}

\begin{abstract}
The college teachers have been aware of the industry requirements of students such as knowledge of latest engineering developments, ethical practices, good communication and team play, flexible and lifelong learning and multitasking. However, a neglected aspect by the teachers, but essential for the industry, is their role in the development of critical thinking skills in the students. In particular, the students need to be made aware of numerous errors of the intellect while still at the college. Published literature on Engineering Education has missed this important aspect. The author recollects what his teacher taught him fifty five years ago at the Indian Institute of Technology, Bombay where he was a student. The memories are supplemented with information and examples from a later period.
\end{abstract}

Keywords: Errors of the intellect, reasoning skills

\section{Introduction}

Drawing conclusions from insufficient data, jumping to conclusions and then finding supporting information instead of the other way, invalid

\section{Balakrishna Palanki}

Mahaveer Educational Society, Bandlaguda, Keshavgiri Post, Hyderabad 500002, India

palankibalakrishna@yahoo.com arguments and inaccurate deductions, omitting information that does not support the hypothesis, are some examples of the errors of the intellect. A glance at some of the project reports being submitted by students of engineering reveals the commission of such errors. This has adversely affected the quality and usefulness of the project reports of the average student. The same errors are likely to be repeated after securing employment in the industry. The intention of the author is to raise the awareness of teachers who would, in turn, attempt to render students more industry ready where analytical skills are needed to resolve issues and for trouble shooting. Students sensitized with the errors of the intellect will avoid them while serving in the industry. This issue has not been addressed so far in the vast literature on engineering education. This paper is expected to trigger systematic and structured research on this aspect of engineering education.

The errors of the intellect have varied in extent and complexity in different branches of engineering. Referring to errors in specific project reports could be controversial. Hence only general examples have been given here that would be understandable by any teacher or student, irrespective of his specialization. History is full of errors of the intellect that resulted in wars, bridge collapses and miscarriage of justice among other things. In modern times too, these errors are being committed in recruitment of personnel, budget allocations, trade off choices, midcourse corrections etc. Errors of the intellect influence engineering judgement and capable of changing the course of a project or organization for the worse. The 
engineers have to be made aware of these early while they are students. This author had the opportunity to witness several errors of the intellect in the students while assessing the project reports. In a previous paper, he dealt with the motivational aspects of teaching and learning at the college [1]. In this paper the focus is on the need to instill critical thinking skills in the students by drawing their attention to the errors of the intellect, so that their contributions and decisions would be free from errors.

\section{Industry Requirements}

It is well known that the industry needs not just followers of algorithms but people who are keen to change the algorithms for the better. People are not judged just by the quantum of production achieved but by the improvements and innovations brought about at the workplace. The industry needs people who are willing to tread new paths to find ways to minimize waste and reduce cost of poor quality. There are several occasions when the go-getter has to take a decision or make a judgement. These call for a good understanding of logic, arguments and the errors of the intellect.

\section{Reasoning skills}

Reasoning skills enable the students and the teachers to perceive patterns and structures in subject information. The patterns help not only in retaining the knowledge from the subject, but also in application of the knowledge in other contexts. It will be useful to understand and critically evaluate different world views. These skills are also handy in the processes of experiment, observation and inference. If the experimental data is wrong, the conclusion is useless, even if valid. It is our ethical responsibility to present both truth and validity. They are essential in formulating arguments and testing of hypotheses. The essential tools such as deductive arguments and inductive arguments facilitate the establishment of cause and effect relationships. Once the basis for the relationships is assimilated, it becomes possible to tailor intellectual as well as material processes with a high benefit to effort ratio. A bird's eye view of the aspects of reasoning are presented, for example by Hank Green [2-4].

\section{Errors of the Intellect}

Plato (424-348 BC) proposed the Tripartite Soul, consisting of the Physical, the Emotional and the
Logical aspects of human beings. The physical aspect drives us to eat, sleep and protect ourselves. In the emotional aspect, feelings fuel our actions. The logical aspect is responsible for innovation and creativity. If the student is made aware of these, he would try to ensure that the third aspect is not unduly influenced by the other two aspects.

Most of the information on errors of the intellect given in this section is from the Indian Institute of Technology, Bombay in the 1960s [5] where this author was a student, though some of the examples emanate from a much later time frame.

\section{Error of ignorance}

This type of error was identified by Plato: "When a person supposes that he knows, and does not know, this appears to be the great source of all the errors of the intellect". In the industry, value is added horizontally whereas control is exercised vertically. So when pockets of ignorance are present in the vertical control, innovative ideas are lost to the detriment of the industry.

\section{Error of coincidence}

It is also known as 'post hoc, ergo propter hoc' from the Latin ("after this, therefore because of this"). It is a fallacy that states: "Since event Y followed event X, event $Y$ must have been caused by event X." When two separate events take place at the same time, one event may be erroneously believed to be the cause and the other as the effect. In Sanskrit, it is called 'Kakataleeyam', which refers to linking of the falling of palm tree branch with the landing of a crow on the branch. The error occurs, for example, in experimental work and forensic analysis. A researchers may find that the topic being investigated by him is also being investigated elsewhere independently by another researcher, and the error of coincidence may be suspected as plagiarism.

\section{Error of the bandwagon}

The term bandwagon refers to accepting or rejecting an argument based on peer pressure. "Many people buy extended warranties. Therefore, it is wise to buy them". "My team holds this as truth. Therefore, everyone who disagrees is wrong". It has been said that there is no trader who would not praise the greatness of his wares. The ideas of the author are great. But his document must list the limitations as 
well. Though the author of the document is great, yet, before approving as reviewer, one must read fully, assess independently, because his signature is his word!

\section{Error of proximity}

The Challenger space flight of 1986 was already postponed several times. Yet, the engineers warned of severe weather conditions and sought postponement again. Having postponed several times in the past, someone in the hierarchy was not inclined to postpone once again. The error of proximity was committed. Low temperature resulted in brittle failure of the rubber gasket leading to fuel leakage through unfilled gaps and explosion. Seven precious lives were lost.

Another Example: The employer want A grade candidates (on the basis of interview) to be recruited, but could not find them in the interview for the whole day. After rejecting several A- candidates, the tired employer selected a B+ candidate. Similarly, after passing several candidates in succession, the examiner may commit the error of proximity by failing the next student in the viva voce examination.

\section{Error of habit}

The story goes that a man on the sea shore was picking up and throwing pebbles into the sea and this soon became a habit. So when he inadvertently picked up a pearl, he threw that as well into the sea. Similarly, when the editor of a technical journal has been seeing and rejecting several ordinary submissions, he may tend to reject a quality submission as well.

\section{Error of familiarity}

It is the space shuttle, once again! Columbia in 2003. The insulating foam that prevented heat from entering shuttle was dislodged making a hole into the cabin. The problem was known in the previous missions, but not fixed. People became familiar with the issue and the concern ebbed down. The defect remained until the disaster. The error of familiarity jeopardized Safety.

If the street is kept clean for most of the time, and then someone throws trash, it raises eyebrows. But if the trashing becomes an everyday affair, people get used to it.
Error of analogy

Let us consider one analogy from the preacher. When the overhead tank contains purified water, the tap delivers pure water. Similarly, when the mind is filled with pure thoughts, the mouth delivers sweet words.

In the subject of heat transfer, the analogy of bricks is often used to explain conduction, convection and radiation. Bricks can be transported from one place to another in three ways - (i) by passing on the brick to the next person in line (conduction), (ii) by carrying the brick from one place to another (convection) and (iii) by throwing from one place to another (radiation). The analogy helps understand but does not prove any argument.

Error of the senses

Every day, the Sun rises in the East, travels above us and finally sets in the West. The movement of the Sun around the earth is so obvious to our senses. Yet we know that it is false. Hence let us not depend on the senses, let us use appropriate instruments.

The Hubble Telescope has shown us distant galaxies. All powders look alike to the naked eye. However, the differences are visible under the microscope.

Are we using the right instruments and sensors in our designs and processes and adhering to procedures?

\section{Error of generalization}

The blind men were examining the elephant by touching. The man who felt the tail said the elephant resembled a rope. The man who touched the ear said it was like a fan. The man who touched the trunk said it was like a pillar. The error of generalization was committed. If a man walks through a town for the first time and sees ten people and all of them happen to be children, he might conclude that all the town's residents are children. Beware of inferences from limited experiments. An example is that of observing a one gram sample of a material and concluding about the one ton lot. The error may be minimized by sharing information, discussion and synthesis of diverse ideas. 
Error of subjectivity

It is frequently applicable that the objectivity gets enhanced when an issue is considered by a group of experts instead of a single individual. The accountability of the group is likely to be greater than that of an individual. Crucial decisions are taken by brainstorming and group discussion to circumvent subjectivity.

\section{Error of irrelevance}

When a public hearing is going on in connection with the proposal to set up a nuclear power station, an activist opposing it will frighten the audience by recollecting the horrors of atomic bombing of Hiroshima and Nagasaki though it is irrelevant to the issue under consideration.

When the student or engineer is making the presentation of a solution to a problem, his critics will mention other problems needing attention to divert the attention of the audience from the current issue.

\section{Error of the machine}

Frequently, students use computer models without fully understanding the algorithm on which the model is based and draw erroneous inferences.

\section{Resistance to Change}

Some cases from history are recollected as examples of resistance to change. Long ago someone claimed he would move a cab without horses. It was the debut for the Internal Combustion Engine. People laughed and threw rotten eggs at him with derision. It was the same with aero planes. Lord Kelvin (18241907) had asserted: "It is thermodynamically impossible for an object heavier than air to fly". The Wright brothers achieved it in 1903 . We would like to remain in our comfort zone and continue to do the way we have always been doing. $100 \%$ safety with $0 \%$ activity! 'A' proposes a change, and 'B' vetoes it. People listen to refute an idea instead of understanding it. It has been proved time and again: What was not possible yesterday will be possible today or tomorrow. Hence let us not nip a new idea in the bud, we will give it a fair trial.

\section{Procrastination and Incubation}

According to Parkinson's Law, "Work expands to fill the time available". It is essential to assign a deadline for every task. This is applicable to students, teachers, heads of departments and all others. A deadline has to be set even for personal tasks. Without a deadline, a job never gets done.

Quite often, the actual time taken for a task is longer than that estimated. In any plan, a cushion period may be provided to absorb the time lost due to unexpected developments.

When a new task arises, the time spent without action is called 'incubation period'. When the engineer receives a purchase file with quotations, he incubates it sufficiently before attempting to make a comparative statement. It is the prerogative of the approving authority as well to incubate the submission further. Such delays result in retendering and additional cost to the organization.

Whether it is for a speech or a power point presentation, it is important to make the first draft as early as possible, well before the deadline. The human mind is ingenious and a new idea flashes every day and the draft can be honed to the highest quality by the time it is due. If time is lost in procrastination, this grand opportunity to refine is lost forever.

\section{In the groove and out of it}

When one first learns to drive the car, one carefully remembers which the brake pedal is and which the clutch release or accelerator pedal is. But with some practice, it becomes a habit and one is able to accelerate, change gears or brake with ease as needed without having to exert mentally. The human mind is wired to form a habit. Once one is habituated to the use of the pedals, one is able to focus on other things on the road. Habit is useful in that it conserves energy of the intellect for higher order tasks. But paradoxically, it is also the habit that prevents one from bringing improvements at the workplace. This has been brought out by Mike Rother very effectively [6].

A child resists when it is told to brush. An adult who is used to brushing his teeth with the right hand will feel uncomfortable when required to do so with the left hand. Similar is the case while having to sign with the other hand than the one normally used. When a student is asked what comes next in $2,4,6,8$, he is likely to say 10 , because the mind is habituated to the existing pattern and jumps to the conclusion of 10 . However, it is also possible that the 8 may be followed by 2 in a cyclic repetition. 
The ox goes round and round in a traditional oil extraction mill. Similarly, people find satisfaction in performing routine work. The teacher must prepare the mind of the student to be able to break the habit and think about new possibilities. It is like driving down to a new place every day prepared for unfamiliar routs. Such a preparedness is essential to bring about changes for the better. An engineering student prepared for change and prepared to change will be undoubtedly industry ready.

\section{Summary}

This paper deals with a topic that has not yet been explored in the field of Engineering Education as seen from a quick scan of the vast published literature. It is expected to be the forerunner of systematic and structured research by eminent educationists. Periodic updating the syllabus by the engineering teacher to meet industry requirement for students is not sufficient in this age of rapid technological changes and information explosion. The college can do its bit by promoting a spirit of inquiry in an environment of choices and collaboration. In particular, making the students aware of the common errors of the intellect is expected to help their critical thinking. There is no substitute for trying, experimentation and critical evaluation of various possible options. In spite of the enforced rules, regulations and adherence to standard practices from the management, affiliating university, State and Federal regulators, the engineering teacher does have freedom to innovate in his teaching methodologies. A student with enlightened exposure at the college will be able to guard himself against the errors of the intellect while serving in the industry. He will create value to the customer and prosperity to the industry. The benefits to the industry spill over to personal and community life as well.

\section{Acknowledgements}

The author thanks Dr. P. Suryanarayana, former Dean, Maulana Azad National Institute of Technology (MANIT), Bhopal for reviewing this paper.

\section{References}

[1] Balakrishna Palanki, (2018) Inspired Teaching and Learning at the Educational Institution and in Industry-Experiments, Experiences and Inferences, Journal of Engineering Education Transformations 31 (3), 215-221.

[2] What is Philosophy?: Crash Course Philosophy \# 1, Hank Green, S quares pa c e https://www.youtube.com/watch?v=1A_CAkYt3 GY

[3] How to Argue - Philosophical Reasoning: Crash Course Philosophy \#2, Hank Green, Squarespace https://www.youtube.com/watch? $\mathrm{v}=\mathrm{NKEhdsnK}$ KHs

[4] How to Argue - Induction \& Abduction: Crash Course Philosophy \#3, Hank Green, Squarespace https://www.youtube.com/watch?v=wrCpLJ1XAw

[5] 2nd B. Tech and 3rd B. Tech Lectures by Mrs. Nalini Swamidasan (1930-2018), Department of Humanities, Indian Institute of Technology, Bombay, teacher of Logic, Economics, Ethics and Social Philosophy from 1962 to 1990.

[6] Mike Rother, Lean Summit 2012, Toyota Kata https://www.youtube.com/watch? $\mathrm{v}=\mathrm{fYoppX} 3 \mathrm{VH}$ IY 